\title{
Study on the Mechanism of Cold and Negative Temperature Coefficient in Natural Process of Gasoline Hydrocarbon
}

\author{
Qu Yakun , Long Jun , Zhou Han \\ Research Institute of Petroleum Processing,SINOPEC, 100083 Beijing,China
}

Email: yakun2015@126.com,longjun2002@126.com

\begin{abstract}
In this paper, the stoichiometric mechanism of gas phase oxidation process of gasoline hydrocarbons was studied through using theoretical stoichiometry. The reason of the phenomenon of cold flame and negative temperature coefficient in the reaction of hydrocarbon molecules before the flame was explained from the molecular level. During the gas phase oxidation process, the alkoxy radical $\mathrm{RO} \cdot$ reacts with hydroxyl $\cdot \mathrm{OH}$ to form a relatively stable intermediate such as aldehyde (or ketone) and $\mathrm{H}_{2} \mathrm{O}$ molecules, and the free radical chain reaction process. The temperature of the reaction process is very low, while the release of a large number of heat, the formation of aldehydes (or ketones) from the excited state back to the ground state when the emission of about $400 \mathrm{~nm}$ wavelength of light blue fluorescence.
\end{abstract}

\section{Introduction}

According to the working principle of the ignition engine, one of the important ways to improve the engine power and reduce the fuel consumption is to improve the engine compression ratio. But with the increase of the engine compression ratio, in terms of fuel, it should be noted that the tendency of the fuel to generate knocking is increased simultaneous [1]. Therefore, the knocking phenomenon became a huge obstacle in the development and use of ignition engines in recent years. The knocking phenomenon in the ignition engine is the abnormal combustion caused by spontaneous combustion of unburned mixture when the normal flame has not yet reached, which is closely related to the high phase oxidation characteristics of the hydrocarbons. From the point of view of oxidation kinetics, the vapor-phase oxidation of hydrocarbons is often characterized by cold flame, that is, weak glow in the phase of gas phase oxidation of hydrocarbon molecules, and the negative temperature coefficient will be occured, that is, the higher the initial temperature, the ignition delay time will be longer, as shown in Figure1. These phenomenons are one of the important characteristics of hydrocarbon gas phase oxidation. According to the experimental observation of many researchers, in addition to methane, methanol, formaldehyde, ethylene and benzene a few hydrocarbon molecules, almost all air heating oxidation of the hydrocarbons will produces cold flame and NCT phenomenon. And the temperature of cold flame produced by the spontaneous combustion process of hydrocarbon molecules has the following regularity: With the increase of carbon atoms in the hydrocarbon molecules, the temperature of the cold flame is significantly reduced; the olefin, naphthenes and aromatic hydrocarbons produce relatively high temperature cold flame, which is very similar to the tendency of generate knocking in the ignition engine.

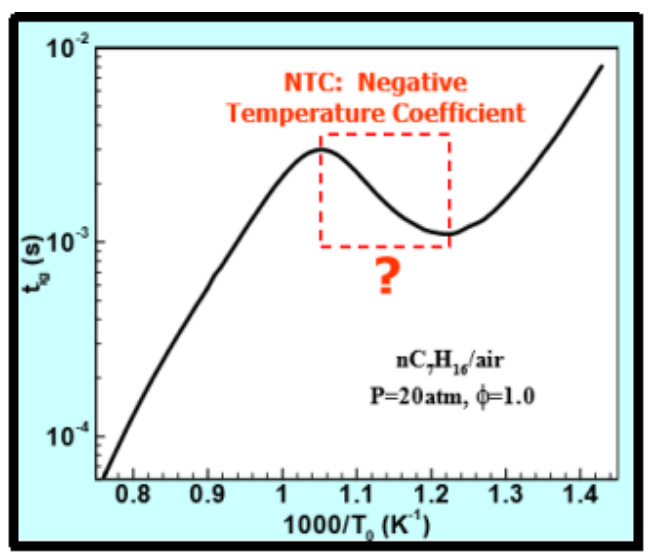

Figure 1. Negative temperature coefficient (NTC) in the process of hydrocarbon spontaneous combustion

With the rapid development of high-precision analysis and characterization technology, the current understanding of finished gasoline has risen to the molecular level, the components of finished gasoline is more than 300 kinds [2]. The range of hydrocarbon molecules is from 4 to 12 , and the temperature range is 
from $30^{\circ} \mathrm{C}$ to $220^{\circ} \mathrm{C}$, including straight and isoparaffins, naphthenes, olefins, aromatic hydrocarbons and oxygen-containing agents[3]. It is the core content of the basic research of combustion physics to study the various elementary reactions and reaction rates occurring at the molecular level of gasoline hydrocarbons [4]. However, recent research works are focused on the in-cylinder physical processes, such as the improvement and improvement of spray, evaporation, and turbulence models, and the importance of fuel chemistry are ignored. Molecular details of chemical composition such as fuel composition and molecular structure, depth understanding combustion mechanism are also lacked, thus the feasibility of controlling combustion and optimizing the concept of combustion from a chemical reaction point of view are limited. Based on the above background, in the paper, it adopts the theoretical chemistry method to study the pre-flame reaction mechanism of the gas phase oxidation process of gasoline $\mathrm{C}_{8}$ hydrocarbon molecules, find out the reason of cold flame and negative temperature coefficient in the reaction of hydrocarbon molecules before the molecular level. It has great important significance on essence of the cause of knocking, the oil refining and engine design.

\section{Calculation Method and Model}

Due to the complexity and volatility of the actual fuels, commercial fuels (gasoline, diesel, kerosene) contain hundreds or more hydrocarbon mixtures. Although advanced chemical analysis can express quantification of the constituent information, and it is difficult to accurately characterize the actual fuel [5]. In order to simulate the physical and chemical properties of the actual fuel, it selected the $\mathrm{C}_{8}$ alkane octadecane octane number of which is -17 . And the initial molecular model of the monomer hydrocarbon is established through referring to the molecular structure parameters in the NIST calculation and the reference database (http//cccbdb.nist.Gov). The structural information is as shown in Figure 2.

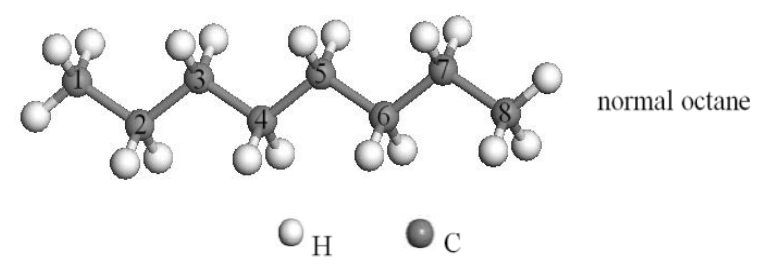

Figure 2. Molecular structure of model compounds

Under the premise of ensuring the accuracy of calculation, the calculation efficiency is selected, and the DFT based on the quantum chemical calculation method is selected. The hybrid function B3LYP $[6,7]$, the wave function is developed in the form of triplet plus polar group TNP (Triple Numerical plus polarization), and the electron treatment is all electronically.

The transitional state search, geometric optimization, and other electronic properties are calculated through using the $\mathrm{DMol}^{3}$ module in the BIOVIA Materials Studio 2017 R2 software package [8]. Fragment analysis, energy decomposition, Hirshfeld charge analysis of the shell system are based on the ADF module [9, 10]. And the calculation of high-spin state of the open shell adopts BAND module[11-14].

In the transition state search, the Linear Synchronous transient and Quadratic Synchronous Transit (LST/QST) method are used and the RMS convergence criterion is $0.002 \mathrm{Ha} / \AA$. The structural optimization convergence criterion is $1 \times 10^{-7} \mathrm{Ha}$, the maximum force is $1 \times 10^{-6} \mathrm{Ha} / \AA$, the maximum displacement is $1 \times 10^{-6} \AA$, and the frequency analysis shows no virtual frequency.

The long-range interaction of $\mathrm{DFT}+\mathrm{D}$ is calculated by TS method. The convergence of self-consistent field SCF (self-consistent field) is accelerated by DIIS method. The multi-pole expansion of density is 16-pole expansion, SCF convergence criterion is $1 \times 10^{-6} \mathrm{Ha}$.

The two fragments of the interaction were analyzed by using the NOCV (Natural orbitals for chemical valence) method and the ETS-NOCV (extended transition state-natural orbitals for chemical valence) method under the Kohn-Sham molecular orbital theory model.

\section{Results and Discussion}

According to the author's previous study on the initiation process of hydrocarbon molecular gas oxidation chain, it is known that the hydrocarbon molecule is inserted into the $\mathrm{C}-\mathrm{H}$ bond through $\mathrm{O}_{2}$ to produce the peroxidase $\mathrm{ROOH}$ of the hydrocarbon.

Then the o-o lysis of peroxide $\mathrm{ROOH}$ causes the hydroxyl radical RO- and hydroxyl - $\mathrm{OH}$ to be generated, as shown in equation (1).

$$
\begin{gathered}
\text { O2 insert C-H key: : } \\
\mathrm{RH}+\mathrm{O} 2 \rightarrow \mathrm{ROOH} \rightarrow \mathrm{RO} \cdot+\cdot \mathrm{OH}
\end{gathered}
$$

In the case of $\mathrm{n}$-octane, the alkoxy radical $\mathrm{C}_{8} \mathrm{H}_{17} \mathrm{O}$ and the hydroxyl $\cdot \mathrm{OH}$ will be produced by the chain initiation process. As shown in Fig.3, the generated alkoxy radical $\mathrm{C}_{8} \mathrm{H}_{17} \mathrm{O}$ can produce $\beta$ cracking at a high temperature, but the reaction activation energy is high, in the hydrocarbon molecular combustion process of the flame before the reaction phase, the temperature is low, such reactions are difficult to occur. At the same time, the formation of the alkoxy radical $\mathrm{C}_{8} \mathrm{H}_{17} \mathrm{O}$ and hydroxyl $\mathrm{OH}$ can occur between the H-reaction, but also with another alkane occurred in the H-reaction. The former product is a more stable compound, which in turn produces a new free radical.

It is well known that the negative temperature coefficient phenomenon occurs during the spontaneous combustion of hydrocarbon molecules which is related to the degradation of the branched chain reaction of the hydrocarbon molecules in the free radical chain reaction, that is, the temporary chain termination characteristic occurs during the reaction. The reaction between the alkoxy radical $\mathrm{C}_{8} \mathrm{H}_{17} \mathrm{O}$ and the hydroxyl $\mathrm{OH}$ produced by the chain initiation has a temporary chain termination property, and the radical reaction can also be considered to belong to the degraded branched chain reaction. The 
activation energy of this reaction is about $0 \mathrm{~kJ} \cdot \mathrm{mol}^{-1}$, the enthalpy becomes $-439.413 \mathrm{~kJ} \cdot \mathrm{mol}^{-1}$, and the activation energy of the reaction is very low, and the reaction is very easy to be studied by quantum chemical calculation based on DFT method. The reaction has characteristic of strong exothermic, so from thermodynamical view is also very easy to be happened.

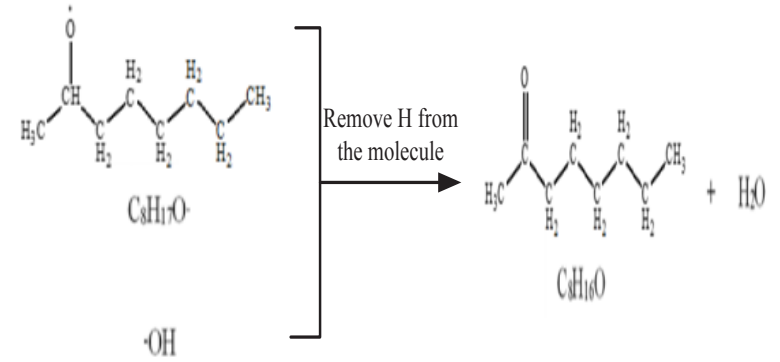

Figure 3. The reaction between free radicals generated by chain initiation

The change of the orbital component of the reaction process is studied based on the molecular orbital theory, as shown in Fig. 4 and Fig. 5. The single occupancy orbital (SMO) of the hydroxyl radicals in the hydroxyl $\mathrm{OH}$ is the $2 \mathrm{px}$ orbital of the $\mathrm{O} \cdot$ atoms in the hydroxyl $\mathrm{OH}$, which is $-8.547 \mathrm{eV}$ after the formation of the $\mathrm{OH}$ and in the presence of the oxygen radical $\mathrm{C}_{8} \mathrm{H}_{17} \mathrm{O}$ (Lowest Unoccupied Molecular Orbital, LUMO) during the reaction which occupied Molecular Orbital, HOMO).

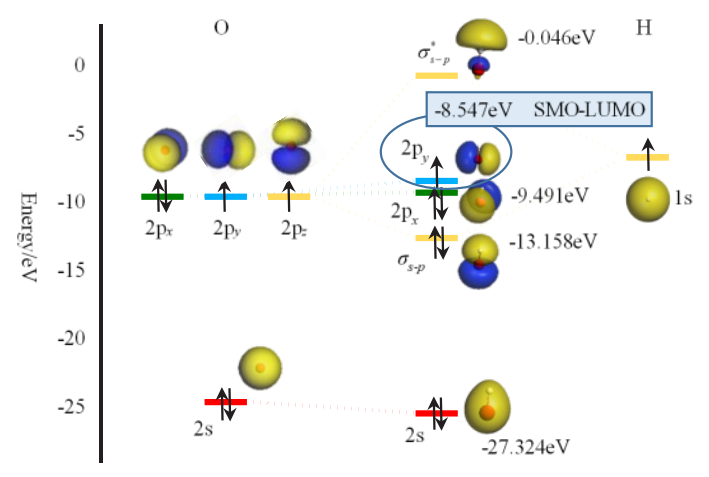

Figure 4. Orbital residence of hydroxyl OH

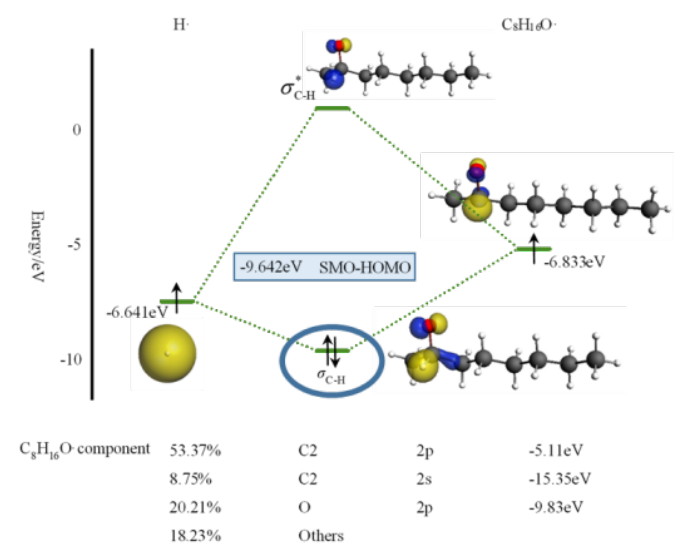

Figure 5. $\mathrm{C}-\mathrm{H}$ bond NOCV in the presence of hydroxyl radical $\mathrm{C}_{8} \mathrm{H}_{17} \mathrm{O}$ with hydroxyl $\mathrm{OH}$ radicals

The $\mathrm{C}-\mathrm{H}$ bond in the $\mathrm{C}_{8} \mathrm{H}_{17} \mathrm{O} \cdot$ participant in the reaction is the $\sigma$ bond formed by the linear combination of the orbital of the $\mathrm{H}$ atom and the 1s orbital of the $\mathrm{H}$ atom, and the formed $\sigma \mathrm{C}-\mathrm{H}$ bonding orbit is affected by the free radical. And the $\mathrm{CH}$ bond is weakened, so that the reaction energy barrier of this reaction is very low. In this paper, the $\pi-\mathrm{C}$ bond is doped into the $2 \mathrm{p}$ orbital component of the $\mathrm{O}$ atom.

During the reaction, electrons are transferred from the $\sigma \mathrm{C}-\mathrm{H}$ bond orbital of the alkoxy radical $\mathrm{C}_{8} \mathrm{H}_{17} \mathrm{O} \cdot$, the highest occupied molecular orbital (HOMO) of the alkoxy radical $\mathrm{C}_{8} \mathrm{H}_{17} \mathrm{O} \cdot$ to the LUMO of hydroxyl $\cdot \mathrm{OH}$.

The reaction of 2-octanone and $\mathrm{H}_{2} \mathrm{O}$ with the reaction of hydroxyl radical $\mathrm{C}_{8} \mathrm{H}_{17} \mathrm{O}$ with hydroxyl $\cdot \mathrm{OH}$ is a relatively stable substance which produces a similar chain termination effect during the vapor phase oxidation of the hydrocarbon molecule. Therefore, it is considered that the reaction is a hydrocarbon molecule The mechanism of NTC phenomenon in the gas phase oxidation process is as shown in Fig.6, which compares the mechanism of the occurrence of NTC phenomenon in the gas phase oxidation process of hydrocarbon molecules and the mechanism found in the study.

$$
\text { Report in other paper }
$$

$$
\text { The Mechanism in this paper }
$$

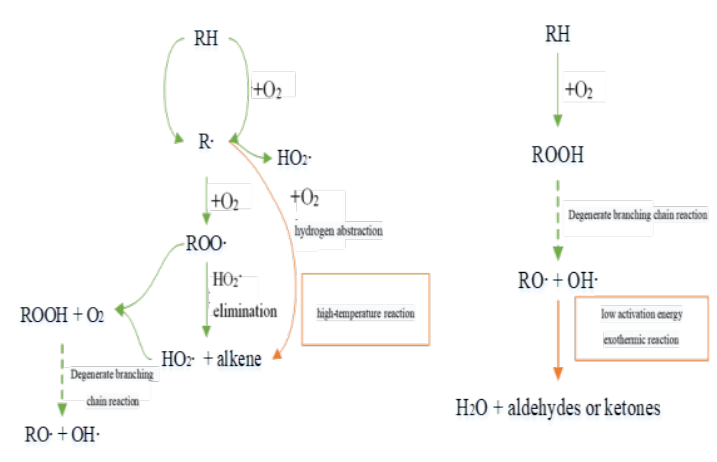

Figure 6. Different mechanisms of NTC phenomenon

The mechanism reported in published literature cannot explain the relationship between the cold flame and the NTC phenomenon comprehensively and cannot explain why the cold flame is produced at the same time, why there is water vapor, which is not consistent with the experimental phenomenon in some parts. In this study, the proposed mechanism of the reaction is not only tan make up for the above problems, but also can find that methane, benzene and other molecules without NTC phenomenon is due to the hydroxyl $\mathrm{OH}$ process, the it first to capture with the oxygen free radicals and $\mathrm{O}$ atoms connected to the $\mathrm{C}$ atom on the $\mathrm{H}$, methane, benzene and other molecular chains caused by the production of alkoxy and $\mathrm{O}$ atoms connected to the $\mathrm{C}$ bond formed by the bond with a strong bond, hydroxyl $\cdot \mathrm{OH}$ reaction activation can be very large, it is difficult to occur this reaction, so the gas phase oxidation process is not cold flame and NTC phenomenon. it explains why there are no cold flame molecules and no NTC phenomenon from the 
perspective of the reaction mechanism.

In the processes of Alkoxy radicals $\mathrm{C}_{8} \mathrm{H}_{17} \mathrm{O}$ and hydroxyl $\cdot \mathrm{OH}$,it can produce some aldehydes and ketones and emit large amounts of heat. Therefore, it is presumed that the glow of the cold flame emitted during the gas phase oxidation of the hydrocarbon molecules is likely to be the oxygen radical $\mathrm{C}_{8} \mathrm{H}_{17} \mathrm{O}$ and in order to further determine the correctness of the reaction mechanism, the hydrogenation of aldehydes or ketones which may be produced by a series of hydrocarbon gas, phase oxidation processes is calculated by the reaction of hydroxyl $\cdot \mathrm{OH}$ to produce aldehydes or ketones excited from the excited state to the ground state. Spectrum is as shown in Fig. 7.

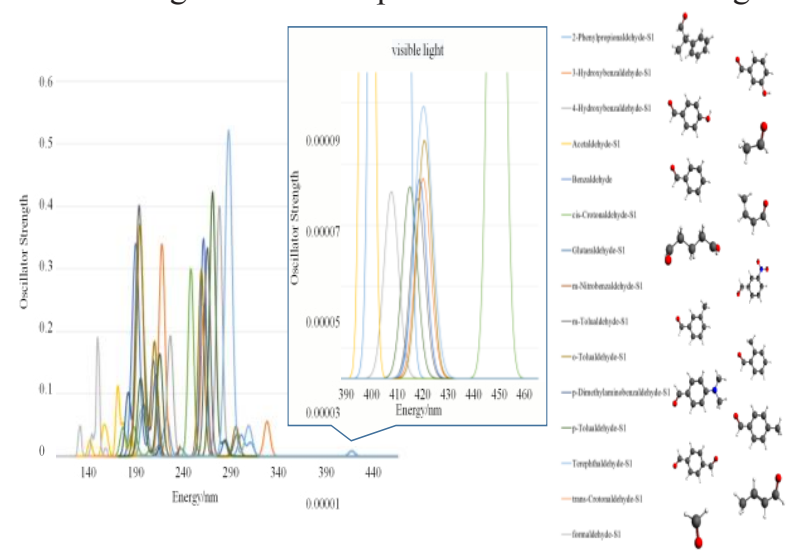

Figure 7. Some aldehydes, ketones and other molecules of the UV absorbable spectrum

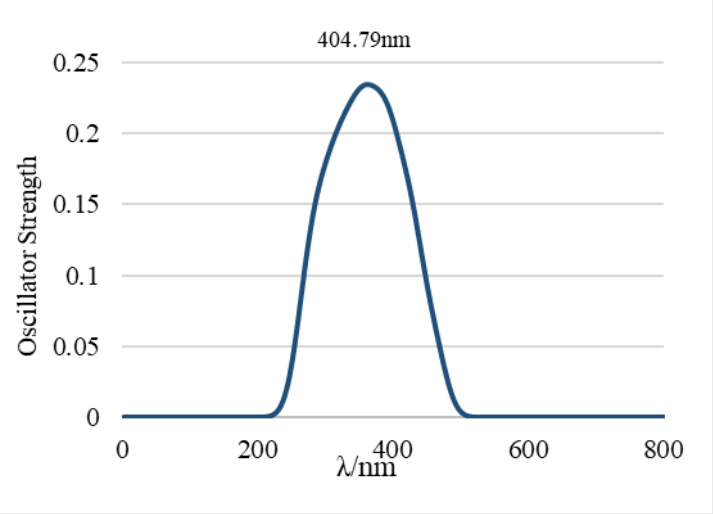

Figure 8. Fluorescence emission spectra of single first excited states of 2-octanone

Generally human eyes can perceive the wavelength of electromagnetic waves between $380 \sim 780 \mathrm{~nm}$, the purple visible wavelength range is about $380 \sim 420 \mathrm{~nm}$, blue visible wavelength is about $440 \sim 475 \mathrm{~nm}$. During the combustion process, Ultraviolet absorption light wavelength of the partial absorption of aldehydes, ketones and other oxides containing the first single excited state of the oxide is about $390 \sim 460 \mathrm{~nm}$, which is basically coincident with the blue-violet wavelengths perceived by the human eye. The glow color released by the cold flame observed by the experiment is also basically coincident. In order to study the chemical nature of the cold flame during the gas phase oxidation of hydrocarbon molecules, the fluorescence emission spectrum of the single first excited state of 2-octanone is calculated. As shown in Fig. 8, the peak wavelength is $404.79 \mathrm{~nm}$, and the excitation energy of the single first excited state of 2-octanone is $295.52 \mathrm{~kJ} \cdot \mathrm{mol}^{-1}$, which is much smaller than that of the reaction. It is further proved that the cold flame produced by the gas phase oxidation of hydrocarbon molecules is the free radicals in the free radical reaction process, the corresponding aldehydes (or ketones) generated by the reaction of the hydroxyl $\cdot \mathrm{OH}$ are excited by the large amount of heat emitted by the reaction, the resulting single first excited state returns to ground state will emit blue violet fluorescence.

\section{Conclusion}

In the process of vapor phase oxidation of hydrocarbons, the oxygen radical $\mathrm{RO} \cdot$ and hydroxyl $\cdot \mathrm{OH}$ are generated by chain initiation, and the reaction between two reactive radicals will form the corresponding derivatives of aldehydes, ketones or other hydrocarbons and $\mathrm{H}_{2} \mathrm{O}$ molecules, the energy barrier of the reaction process is very low, and will approach 0 , and a large amount of heat is released, so that the generated aldehyde or ketone is excited from the ground state to the single first excited state, and when it returns from the excited state to the ground state, it will emits about $400 \mathrm{~nm}$ blue-violet fluorescence.

In the process of gas phase oxidation of hydrocarbons, in the progress of the reaction, the temperature will be risen, the reaction will be slow, the main reason is the chain reaction lead to produce oxygen radical RO and hydroxyl $\cdot \mathrm{OH}$ and to produce a corresponding halide, ketone or other hydrocarbon derivatives and $\mathrm{H}_{2} \mathrm{O}$ molecules and other relatively stable intermediates, which cause vapor phase oxidation of hydrocarbons free radical chain reaction process temporarily terminated.

\section{References}

1. Wang Ning and Wen Hao, the chemical composition and structure of gasoline fuel and the relationship between the structure and anti-explosive properties of the oil refining and chemical industry, 27 (2),41-46(1996)

2. Liu Yuhua, Ma Wenyou, Full Analysis of Monomer Hydrocarbons in Catalytic Cracking Gasoline. Journal of Qiqihar University (Natural Science Edition), (2),68-69(1999)

3. Allison, T.C. Workshop on Combustion Simulation Databases for Real Transportation Fuels. In The Workshop on Combustion Simulation Databases for Real Transportation Fuels,(2003)

4. Liu Zelong. Explore the oil molecules "mettle." Sinopec, (2),50-51(2014)

5. Xiao Gan, Zhang Yusheng, Jiang Guangjun. Systematic Construction and Verification of Gasoline Multi-component Characterization of Fuel Simplification Dynamics Model. Journal of Physical Chemistry, (4), 879-892(2016) 
6. Becon, A., Density functional thermochemistry. III. The role of exact exchange. J. Chem. Phys, 98, 5648-5652(1993)

7. Stephens, Pauk, Ab initio calculation of vibrational absorption and circular dichroism spectra using density functional force fields. The Journal of Physical Chemistry, 98 (45),11623-11627(1994)

8. Delley, B., DMOL is a density functional theory (DFT) program distributed by Accelrys, Inc. J. Chem. Phys, 92(1990)

9. Te Velde, G.t., Chemistry with ADF. Journal of Computational Chemistry, 22 (9), 931-967(2001)

10. Guerra, C.F., Towards an order-N DFT method. Theoretical Chemistry Accounts,99(6),
11. Te Velde, G. and E. Baerends, Precise density-functional method for periodic structures. Physical Review B, 44 (15), 7888(1991).

12. Wiesenekker, G. and E. Baerends, Quadratic integration over the three-dimensional Brillouin zone. Journal of Physics: Condensed Matter, 3 (35), 6721(1991).

13. Franchini, M., P.H.T. Philipsen, and L. Visscher, The Beckm fuzzy cells integration scheme in the Amsterdam Density Functional program suite. Journal of computational chemistry,34(21), 1819-1827(2013).

14. Franchini, M., Accurate Coulomb Potentials for Periodic and Molecular Systems through Density Fitting. Journal of Chemical Theory and Computation, 10 (5), 1994-2004(2014). 